Sitientibus Série Ciências Físicas 16, sf01608-1 (2020)

\title{
Preparation of a Superposition of Squeezed Coherent States of a Cavity Field via Coupling to a Superconducting Charge Qubit
}

\section{Preparação de uma Superposição de Estados Coerentes Comprimidos de um Campo na Cavidade Acoplado a um Qubit de Carga Supercondutora}

\author{
Dagoberto S. Freitas* \\ Departamento de Física - UEFS \\ Campus UEFS, Novo Horizonte, \\ Feira de Santana - BA - 44036-900
}

\begin{abstract}
The generation of nonclassical states of a radiation field has become increasingly important in the past years given its various applications in quantum communication. It has been recently proposed a way to engineer quantum states using a SQUID charge qubit inside a cavity with a controllable interaction between the cavity field and the charge qubit. Since decoherence is known to affect quantum effects uninterruptedly and decoherence process are working even when the quantum state is being formed, therefore, is interesting to envisage processes through which quantum superpositions are generated as fast as possible. We succeed in linearizing the Hamiltonian of the system through the application of an appropriate unitary transformation and for certain values of the parameters involved, we show that it is possible to obtain specific Hamiltonians. In this work we will use this approach for preparing superposition of two squeezed coherent states.
\end{abstract}

Keywords: Superposition states, Squeezed coherent states, Superconduting charge qubit.

Nos últimos anos, devido às suas várias aplicações na comunicação quântica a geração de estados quânticos de um campo de radiação tem se tornado cada vez mais importante. Recentemente, foi proposta uma maneira de construir estados quânticos usando um qubit de carga (SQUID) dentro de uma cavidade com uma interação controlável entre o campo da cavidade e o qubit. Na geração de estados quânticos o processo de decoerência é conhecido por afetar os efeitos quânticos de forma ininterrupta, mesmo quando o estado quântico está sendo formado. Dessa forma, é importante imaginar processos através dos quais as superposições quânticas são formadas o mais rápido possível. Através da linearização do Hamiltoniano do sistema, utilizando uma transformção unitária apropriada, e para certos valores dos parâmetros envolvidos, mostramos que é possível obter Hamiltonianos que levam a processos mais rápidos de geração de estados quânticos. Neste trabalho, usaremos essa abordagem para preparar a superposição de dois estados coerentes comprimidos.

Palavras-chaves: Superposição de estados, Estados coerentes comprimidos, Qubit de carga supercondutor.

\section{INTRODUCTION}

In the past years, the generation of nonclassical states of a radiation field has become more and more important, thanks to its applications in quantum communication. The possibility of generating nonclassical states has been possible

*E-mail: dfreitas@uefs.br in various branches of physics, such as cavity electrodynamics, trapped ions, quantum dots, atoms within cavities, and so on [1, 2]. In this sense, we will discuss the generation of nonclassical states in the context of a superconducting qubit in a microcavity. Recently, it was proposed a way to project quantum states using a qubit (SQUID) charge qubit inside a cavity [5, 6] with a controllable interaction between the cavity field and the charge qubit. 
The main ingredients for projecting quantum states are a tunable gate voltage and a classic magnetic field applied to SQUID. The recent interest in the study of the cavity quantum electrodynamics type systems such as a superconducting qubit can open new ways for studying the interaction between light and solid-state quantum devices [3, 4]. Various theoretical and experimental works have discussed the interaction between superconducting qubits with either quantized [5] 19] or classical fields [20 22]. In references [5, 6] the model proposed including these ingredients and using some adequate approximations which allow for the linearization of the interaction and nonclassical states of the field are generated.

The single-cavity scheme of reference [5, 6] may be extended to generate entangled coherent states of two microwave cavity fields coupled to a SQUID-type superconducting box, as proposed in reference [8]. In the literature it can also be found proposals for the generation of entangled states and squeezed states using linear and nonlinear interactions between microwave cavity field and SQUID-type superconducting box [9]; schemes for generation of multi-qubit entangled cluster states [10, for deterministic generation of entangled photon pairs in a superconducting resonator array [11, and for controlling the entanglement between two Josephson charge qubits [12].

We show that the essential contribution of the nonlinear interaction is to shorten the time necessary to build the quantum state. Since decoherence is known to affect quantum effects uninterruptedly, they are at work even while the quantum state is being formed. This has been studied and quantified in the context of cavity QED where it is shown that the more quantum is the superposition the more rapid are the environmental effects during the process of creating the quantum state [23]. It is therefore interesting to envisage processes through which quantum superpositions are generated as fast as possible.

\section{THE MODEL}

We consider a system constituted by a SQUID type superconducting box with $n_{c}$ excess Cooper-pair charges connected to a superconducting loop via two identical Josephson junctions having capacitors $C_{J}$ and coupling energies $E_{J}$, see Fig.(17). An external control voltage $V_{g}$ couples to the box via a capacitor $C_{g}$. We also assume that the system operates in a regime consistent with most experiments involving charge qubits, in which only Cooper pairs coherently tunnel in the superconducting junctions. Therefore, the system Hamiltonian may be written as $[5,6,24$.

$$
H_{q b}=4 E_{c h}\left(n_{c}-n_{g}\right)^{2}-2 E_{J} \cos \left(\frac{\pi \Phi_{X}}{\Phi_{0}}\right) \cos (\Theta),
$$

where $E_{c h}=e^{2} / 2\left(C_{g}+2 C_{J}\right)$ is the singleelectron charging energy, $n_{g}=C_{g} V_{g} / 2 e$ is the dimensionless gate charge (controlled by $V_{g}$ ), $\Phi_{X}$ is the total flux through the SQUID loop and $\Phi_{0}$ the quantum flux. The phase $\Theta$ is the quantum-mechanical conjugate of the number operator $n_{c}$ of the Cooper pairs in the box. The superconducting box is assumed to be working in the charging regime and the superconducting energy gap $\Delta$ is considered to be the largest energy involved. Moreover, the temperature $T$ is low enough so that $\Delta \gg E_{c h} \gg E_{J} \gg k_{B} T$, where $k_{B}$ is the Boltzmann constant.

The superconducting box then becomes a two-level system with states $|g\rangle\left(\right.$ for $\left.n_{c}=0\right)$ and $|e\rangle$ (for $n_{c}=1$ ) given that the gate voltage is near a degeneracy point $\left(n_{g}=1 / 2\right)$ 24] and the quasi-particle excitation is completely suppressed [25].

If the circuit is placed within a single-mode microwave superconducting cavity, the qubit can be coupled to both a classical magnetic field (generates a flux $\Phi_{c}$ ) and the quantized cavity field (generates a flux $\Phi_{q}=\eta a+\eta^{*} a^{\dagger}$, with $a$ and $a^{\dagger}$ the annihilation and creation operators), being the total flux through the SQUID given by $\Phi_{X}=\Phi_{c}+\Phi_{q}$ [7], see Fig. (1). The parameter $\eta$ is related to the mode function of the cavity field. 
The Hamiltonian system will then read

$H=\hbar \omega a^{\dagger} a+E_{z} \sigma_{z}-E_{J} \sigma_{x} \cos \left(\gamma I+\beta a+\beta^{*} a^{\dagger}\right)$,

where we have defined the parameters $\gamma=$ $\pi \Phi_{c} / \Phi_{0}$ and $\beta=\pi \eta / \Phi_{0}$. The first term corresponds to the free cavity field with frequency $\omega=4 E_{c h} / \hbar$ and the second one to the qubit having energy $E_{z}=-2 E_{c h}\left(1-2 n_{g}\right)$ with $\sigma_{z}$ and $\sigma_{x}$ the Pauli matrices. The third term is the (nonlinear) photon-qubit interaction which may be controlled by the classical flux $\Phi_{c}$.

(a)
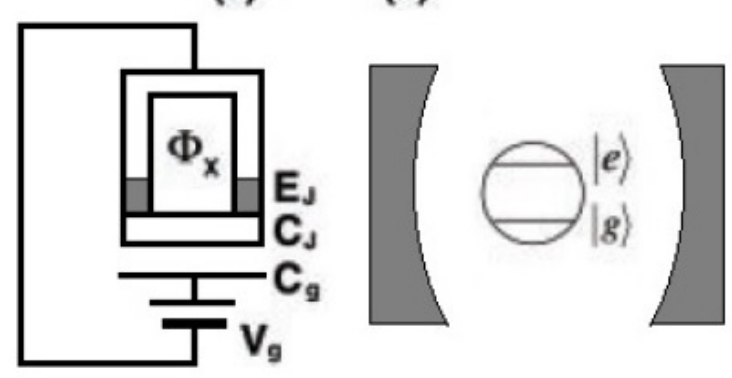

FIG. 1: (a) A charge qubit formed by a SQUID device, equivalent to a controllable macroscopic two-level system, is placed into a superconducting microwave cavity in (b). The coupling between the quantized cavity field and qubit system is realized via the magnetic flux $\Phi_{X}$ through the SQUID. Figure adapted from reference [26].

In general the Hamiltonian in equation (2) is linearized under some kind of assumption. In Ref. [5, 6], for instance, the authors decomposed the cosine in Eq. (2) and expanded the terms $\sin \left[\pi(\eta a+H . c.) / \Phi_{0}\right]$ and $\cos [\pi(\eta a+$ H.c. $\left.) / \Phi_{0}\right]$ as power series in $a\left(a^{\dagger}\right)$. In this way, if the limit $|\beta| \ll 1$ is taken, only singlephoton transition terms in the expansion are kept, and a Jaynes-Cummings type Hamiltonian (JCM) is then obtained. In contrast to that, in the references 27-31 it is presented a technique that obtain a JCM Hamiltonian valid for any value of $|\beta|$. This technique consists in applying an unitary transformation that linearizes the Hamiltonian of the sys- tem. After transforming the original Hamiltonian, it is possible to obtain a simpler Hamiltonian under certain resonance regimes. In other words, it is possible linearize the superconductor/quantized field Hamiltonian without doing the usual power series expansions of the Hamiltonian.

\section{DYNAMICS OF THE SYSTEM}

The central idea of the approach proposed in reference [27] which allows for the inclusion of nonlinear effects is the following: an unitary transformation is constructed in a way that diagonalizes the Hamiltonian leading it to a much simpler form. The nonlinear effects are therefore retained in the transformation affecting directly the time evolution of the system in a tractable manner. The comparison of our proposal with other method is not simple. Normally the full hamiltonian is truncated after some kind of approximation - for instance, by taking the limit $|\beta|<<1$, a simple linearized [6] or nonlinear Hamiltonians [7] are obtained.

In the method used here it is possible to obtain in a direct way, a Hamiltonian which allows an exact solution for the state vector in a specific resonance regime (as long as $|\beta|$ ). However, as the nonlinear effects are somehow retained in the transformed Hamiltonian, they may give rise to a more complex dynamics, for example: in reference [29] for the preparation a Schrödinger cat (SC) of mode of cavity field interacting with a superconducting charge qubit; in reference [30, the resulting dynamics exhibits typical behavior of a driven JaynesCummings model [32] (or a trapped ion within a cavity [27]), but without the presence of a classical driving field; in reference [28] for the preparation of $\mathbf{S C}$ with cold ions.

We believe that the approach used here could be useful not only for establishing a direct connection to other well-known models in quantum optics, but also the exploration of different regimes in superconducting systems. Next we apply a unitary transformation to the full Hamiltonian given by (2) and make approximations afterwards. By applying the unitary 
transformation [27,

$T=\frac{1}{\sqrt{2}}\left\{-\frac{1}{2}\left[D^{\dagger}(\alpha, \gamma)-D(\alpha, \gamma)\right] I-\frac{1}{2}\left[D^{\dagger}(\alpha, \gamma)+D(\alpha, \gamma)\right] \sigma_{z}+D(\alpha, \gamma) \sigma_{+}+D^{\dagger}(\alpha, \gamma) \sigma_{-}\right\}$

to the Hamiltonian in Eq.(2), with $D(\alpha, \gamma)=D(\alpha) e^{i \frac{\gamma}{2}}$ where $D(\alpha)=\exp \left[\left(\alpha a^{\dagger}-\right.\right.$ $\left.\alpha^{*} a\right)$ ] is the Glauber's displacement operator, with $\alpha=i \beta^{*} / 2$, we obtain the following transformed Hamiltonian

$$
\begin{aligned}
H_{T} & \equiv T H T^{\dagger}=\hbar \omega a^{\dagger} a+\frac{E_{J}}{2} \sigma_{z}+i \frac{\hbar}{2}\left[\omega\left(\beta a-\beta^{*} a^{\dagger}\right)+2 i \frac{E_{z}}{\hbar}\right] \sigma_{x}+\frac{E_{J}}{2} \cos \left[2\left(\beta a+\beta^{*} a^{\dagger}\right)+2 \gamma\right] \sigma_{z} \\
& -i \frac{E_{J}}{2} \sin \left[2\left(\beta a+\beta^{*} a^{\dagger}\right)+2 \gamma\right]\left(\sigma_{+}-\sigma_{-}\right)+\left|\frac{\beta}{2}\right|^{2} .
\end{aligned}
$$

This result holds for any value of the parameter $\beta$. In the regime in which $\hbar \omega|\beta|=4|\beta| E_{c h} \gg$ $E_{J}$, that can be obtained for $|\beta| \geq 0.25$, the Hamiltonian in Eq. (4) becomes,

$$
\begin{aligned}
H_{T} & \cong \hbar \omega a^{\dagger} a+\frac{E_{J}}{2} \sigma_{z}+i \frac{\hbar \omega}{2}\left[\left(\beta a-\beta^{*} a^{\dagger}\right)\right. \\
& \left.+2 i \frac{E_{z}}{\hbar \omega}\right] \sigma_{x} .
\end{aligned}
$$

Our Hamiltonian in Eq. (5) becomes a JaynesCummings type Hamiltonian. For $|\beta|=0.25$ the charge regime, $E_{c h} \gg E_{J}$, is satisfied. Note that in the approach of reference [5], the condition $|\beta| \ll 1$ is also necessary, but for a different reason, i.e., to truncate the co-sine (sine) series.

We should remark that in our scheme the Jaynes-Cummings evolution takes place in the transformed frame, differently from the model developed in [5]. The term $\left|\frac{\beta}{2}\right|^{2}$ was not taken into account because it just represents an overall phase. The same setup and transformation given by (3) may also be employed (see reference [29]) in a scheme for preparation of superpositions of coherent states of a single-mode cavity field (Schrödinger cats - SC) extending the approach of reference [5]. The results is very similar to the $\mathbf{S C}$ obtained in reference [5], but in contrast to that we did not use the condition $|\beta| \ll 1$. In our scheme proposed in [29], as $|\beta|$ is large and the value of the amplitude of coherent states are proportional to $t^{2}$, the time for preparing of an observable $\mathbf{S C}$ state is much shorter than the approach adopted in reference [5].

Now we show how to prepare a superposition of two squeezed coherent states. To obtain this superposition we set $E_{z}=0\left(n_{g}=1 / 2\right)$ in (5). Here, we take advantage of the fact that the Hamiltonian given in Eq.(5) has not been approximated and, therefore, there are no restriction on the values of their parameters. By transforming the Hamiltonian (5) with the unitary operators,

$$
U_{1}=e^{\varepsilon_{1}\left(a^{\dagger} \sigma_{+}+\sigma_{-} a\right)},
$$

and

$$
U_{2}=e^{\varepsilon_{2}\left(a \sigma_{+}+\sigma_{-} a^{\dagger}\right)},
$$

with $\varepsilon_{1}, \varepsilon_{2} \ll 1$, and $\sigma_{-}, \sigma_{+}$are the Pauli matrices. Setting as

$$
\varepsilon_{1}=\frac{i \hbar \omega \beta}{2\left(E_{J}-\hbar \omega\right)}
$$

and

$$
\varepsilon_{2}=-\frac{i \hbar \omega \beta}{2\left(E_{J}+\hbar \omega\right)},
$$

where we consider $\beta$ as real. Remaining up to 
first order in the expansion $e^{\varepsilon A} B e^{-\varepsilon A}=B+$ $\varepsilon[A, B]+\frac{\varepsilon^{2}}{2 !}[A,[A, B]]+\cdots \approx B+\varepsilon[A, B]$, i.e., doing a small rotation, we obtain the Hamiltonian,

$$
\begin{aligned}
H_{e f f} & \equiv U_{2} U_{1} H_{T} U_{1}^{\dagger} U_{2}^{\dagger} \\
& =\hbar \omega a^{\dagger} a+\frac{E_{J}}{2} \sigma_{z}-\frac{\left(E_{J}-\hbar \omega\right) \beta^{2} \hbar^{2} \omega^{2}}{4\left(E_{J}^{2}-\hbar^{2} \omega^{2}\right)} \\
& \times\left(a^{2}+a^{\dagger 2}\right) \sigma_{z}+\frac{E_{J} \beta^{2} \hbar^{2} \omega^{2}}{E_{J}^{2}-\hbar^{2} \omega^{2}}\left(a^{\dagger} a+\frac{1}{2}\right) \sigma_{z} .
\end{aligned}
$$

In the Eq.10) the first interaction term describes a squeezed state Hamiltonian and the second interaction one describe a dispersive Hamiltonian. For the regime in which $\hbar \omega-E_{J} \gg 4 E_{J}$ the Hamiltonian in Eq. 10 becomes a squeezed Hamiltonian,

$$
\begin{aligned}
H_{e f f} & =\hbar \omega a^{\dagger} a+\frac{E_{J}}{2} \sigma_{z}-\frac{\left(E_{J}-\hbar \omega\right) \beta^{2} \hbar^{2} \omega^{2}}{4\left(E_{J}^{2}-\hbar^{2} \omega^{2}\right)} \\
& \times\left(a^{2}+a^{\dagger 2}\right) \sigma_{z} .
\end{aligned}
$$

Now, to obtain a superposition of squeezed coherent states we will make a rotation on Eq.(11) so that $\sigma_{z} \rightarrow \sigma_{x}$. This rotation is equivalent to applying the operator,

$$
U_{R}=\exp \left(-i \frac{\pi}{4} \sigma_{y}\right)=\frac{\sqrt{2}}{2}\left[1-\left(\sigma_{+}-\sigma_{-}\right)\right]
$$

on (11) we obtain the Hamiltonian,

$$
\begin{aligned}
H_{S S} & \equiv U_{R} H_{e f f} U_{R}^{\dagger} \\
& =\hbar \omega a^{\dagger} a+\frac{E_{J}}{2} \sigma_{x}-\frac{\left(E_{J}-\hbar \omega\right) \beta^{2} \hbar^{2} \omega^{2}}{4\left(E_{J}^{2}-\hbar^{2} \omega^{2}\right)} \\
& \times\left(a^{2}+a^{\dagger 2}\right) \sigma_{x},
\end{aligned}
$$

with $U_{R} \sigma_{z} U_{R}^{\dagger}=\sigma_{x}$. If the system is initially in the coherent state $|\gamma\rangle$ and the charge qubit is in the ground state $|g\rangle=\frac{1}{\sqrt{2}}(|+\rangle-|-\rangle)$, where $|+\rangle(|-\rangle)$ is eigenstate of the Pauli operator $\sigma_{x}$ with the eigenvalue $1(-1)$, we can entangle qubit states with superpositions of two different squeezed coherent states (SS) evolving in time as,

$$
\left|\Psi_{T}^{R}(t)\right\rangle=U(t)|g\rangle|\gamma\rangle,
$$

where $U(t)=\exp \left(-i \frac{H_{S S}}{\hbar} t\right)$ is the time Evolution operation. The time evolution of the state vector is given by,

$$
\left|\Psi_{T}^{R}(t)\right\rangle=\frac{1}{\sqrt{2}}\left[\left|\Phi(t)_{-}\right\rangle|e\rangle+\left|\Phi(t)_{+}\right\rangle|g\rangle\right],
$$

where the superposition of two squeezed coherent states is,

$$
\begin{aligned}
\left|\Phi(t)_{ \pm}\right\rangle & =\frac{1}{\sqrt{2}}\left[e^{-i \frac{E_{J}}{2} t}\left|\gamma,-i \xi^{2} t / \hbar\right\rangle\right. \\
& \left. \pm e^{i \frac{E_{J}}{2} t}\left|\gamma, i \xi^{2} t / \hbar\right\rangle\right]
\end{aligned}
$$

with $\xi^{2}$ given by,

$$
\xi^{2}=\frac{\beta^{2} \hbar^{2} \omega^{2}}{4\left(E_{J}+\hbar \omega\right)},
$$

and

$$
\left|\gamma, \mp i \xi^{2} t / \hbar\right\rangle=e^{-i \omega a^{\dagger} a t \mp i \xi^{2}\left(a^{2}+a^{\dagger 2}\right) t / \hbar}|\gamma\rangle .
$$

Here, $\left|\gamma, \mp i \xi^{2} t / \hbar\right\rangle$ denote the squeezed coherent states, and the degree of squeezing is determined by the time-dependent parameter:

$$
\xi^{2} t / \hbar=\frac{\beta^{2} \hbar \omega^{2}}{4\left(E_{J}+\hbar \omega\right)} t .
$$

The result is an entangled state involving qubit and a cavity field. If one measures the charge state (either in $|g\rangle$ or $|e\rangle$ ), the action will collapse the $\left|\Psi_{T}^{R}(t)\right\rangle$ into a SS state $\left|\Phi_{ \pm}\right\rangle$. The form of Eq. (15) is very similar to the SS obtained in reference [5]. But, in contrast to that, we did not do use the condition $\beta \ll 1$. In our scheme, as $\beta$ is large, and the value of the amplitude of coherent states are given by $\xi^{2} t / \hbar=\frac{\beta^{2} \hbar \omega^{2}}{4\left(E_{J}+\hbar \omega\right)} t \approx \beta^{2} E_{c h} t / \hbar,\left(\hbar \omega=4 E_{c h}\right.$ and $E_{c h} \gg E_{J}$ ), the time for preparing an observable SS state is much shorter than that in the schemes proposed in [5].

\section{CONCLUSION}

In conclusion, we have presented an approach for preparing SS states of the mode of cavity field interacting with a superconducting charge qubit. In contrast to other schemes we 
include nonlinear effects. In general, approximations are made directly to the full Hamiltonian in Eq.(2) neglecting all higher orders of $\beta$. In our scheme, we first apply an unitary transformation to the Hamiltonian (2) and make the relevant approximations after performing the transformation. The result obtained holds for any value of the parameter $\beta$. In the regime when $\hbar \omega \beta \gg E_{J}$, which it can be obtained for $\beta \geq 0.25$, the Hamiltonian becomes a squeezed Hamiltonian. Based on the measurement of charge states, we show how the SS states of a single-mode cavity field can be generated it. Here, as $|\beta|$ is large, and the amplitude of coherent states are proportional to $\beta^{2} E_{c h} t / \hbar$, the time for preparing observable $\mathbf{S S}$ states is much shorter than that in the linear regimes.
[1] M. Brune, E. Hagley, J. Dreyer, X. Maître, A. Maali, C. Wunderlich, J.M. Raimond, S. Haroche. Phys. Rev. Lett. 77, 4887 (1996).

[2] C. Monroe, D.M. Meekhof, B.E. King, D.J. Wineland. Science 272, 1131 (1996).

[3] I. Buluta, S. Ashhab, F. Nori. Rep. Prog. Phys. 74, 104401 (2011).

[4] Z.-L. Xiang, S. Ashhab, J.Q. You, F. Nori. Rev. Mod. Phys. 85, 623 (2013).

[5] Y. Liu, L.F. Wei, F. Nori. Phys. Rev. A71, 063820 (2005).

[6] Y. Liu, L.F. Wei, F. Nori. Europhys. Lett. 67, 941 (2004).

[7] J.Q. You, F. Nori. Phys. Rev. B68, 064509 (2003).

[8] J.-Q. Liao, L.-M. Kuang. J. Phys. B40, 1845 (2007).

[9] W.Y. Huo, G.L. Long. New Journal of Physics 10, 013026 (2008).

[10] S. Ke-Hui. Chinese Phys. Lett. 26, 120302 (2009).

[11] Y. Hu, L. Tian. Phys. Rev. Lett. 106, 257002 (2011).

[12] L. Qing-Hong, F. Guang-Yu, W. Ji-Cheng, A.M. Ashfaq, L. Shu-Tian. Chinese Phys. Lett. 28, 060307 (2011).

[13] A.M. Zagoskin, M. Grajcar, A.N. Omelyanchouk. Phys. Rev. A70, 060301 (2004).

[14] M. Abdel-Aty. Optics Communications 282, 4556 (2009).

[15] S. Ashhab, F. Nori. Phys. Rev. A81, 042311 (2010).

[16] K.-H. Song, Y.-J. Zhao, Z.-G. Shi, S.-H. Xiang, X.-W. Chen. Optics Communications 283, 506 (2010).

[17] C. Valverde, A.T. Avelar, B. Baseia. Physica A: Statistical Mechanics and its Applications 390, 4045 (2011).
[18] C. Valverde, H.C.B. de Oliveira, A.T. Avelar, B. Baseia. Chinese Phys. Lett. 29, 080303 (2012).

[19] L. Tang, F. Liu. Physics Letters A378, 2074 (2014).

[20] Z. Zhou, S. Chu, S. Han. Phys. Rev. B66, 054527 (2002)

[21] E. Paspalakis, N.J. Kylstra. J. Mod. Opt. 51, 1679 (2004).

[22] Y. Liu, J.Q. You, L.F. Wei, C.P. Sun, F. Nori. Phys. Rev. Lett. 95, 087001 (2005).

[23] J.G. Peixoto, M.C. Nemes. Journal of Optics B5, 265 (2002).

[24] Y. Makhlin, G. Schön, A. Shnirman. Rev. Mod. Phys. 73, 357 (2001).

[25] D.V. Averin, Y.V. Nazarov. Phys. Rev. Lett. 69, 1993 (1992).

[26] Yu-Xi Liu, L. F. Wei, F. Nori. Europhys. Lett. 67, 941 (2004).

[27] H. Moya-Cessa, A. Vidiella-Barranco, J.A. Roversi, D.S. Freitas and S.M. Dutra. Phys. Rev. A59, 2518 (1999).

[28] M. Feng. Phys. Lett. A282, 230 (2001).

[29] D.S. Freitas, M.C. Nemes. Modern Physics Letters B28, 1450082 (2014).

[30] D.S. Freitas, A. Vidiella-Barranco, J.A. Roversi. Eur. Phys. J. D 68, 193 (2014).

[31] H. Moya-Cessa. Sci. Rep. 6, 38961 (2016).

[32] S.M. Dutra, P.L. Knight, H. Moya-Cessa. Phys. Rev. A 49, 1993 (1994).

[33] L. Davidovich, M. Brune, J.M. Raimond, S. Haroche. Phys. Rev. A53, 1295 (1996).

[34] M.C. de Oliveira, M.H.Y. Moussa, S.S. Mizrahi. Phys. Rev. A61, 063809 (2000).

[35] J.M. Raimond, M. Brune, S. Haroche. Rev. Mod. Phys. 73, 565 (2001). 